\title{
Probiotics-induced changes in gut microbial composition and its effects on cognitive performance after stress: exploratory analyses
}

\author{
Mirjam Bloemendaal (10), Joanna Szopinska-Tokov (1)', Clara Belzer², David Boverhoff3', Silvia Papalini,3,4,
} Franziska Michels ${ }^{3}$, Saskia van Hemert $\mathbb{D}^{5}$, Alejandro Arias Vasquez ${ }^{1}$ and Esther Aarts ${ }^{3}$

\begin{abstract}
Stress negatively affects cognitive performance. Probiotics remediate somatic and behavioral stress responses, hypothetically by acting on the gut microbiota. Here, in exploratory analyses, we assessed gut microbial alterations after 28-days supplementation of multi-strain probiotics (EcologicBarrier consisting of Lactobacilli, Lactococci, and Bifidobacteria in healthy, female subjects (probiotics group $n=27$, placebo group $n=29$ ). In an identical pre-session and post-session, subjects performed a working memory task before and after an acute stress intervention. Global gut microbial beta diversity changed over time, but we were not able to detect differences between intervention groups. At the taxonomic level, Time by Intervention interactions were not significant after multiple comparison correction; the relative abundance of eight genera in the probiotics group was higher (uncorrected) relative to the placebo group: Butyricimonas, Parabacteroides, Alistipes, Christensenellaceae_R-7_group, Family_XIII_AD3011_group, Ruminococcaceae_UCG-003, Ruminococcaceae_UCG-005, and Ruminococcaceae_UCG-010. In a second analysis step, association analyses were done only within this selection of microbial genera, revealing the probiotics-induced change in genus Ruminococcaceae_UCG-003 was significantly associated with probiotics' effect on stress-induced working memory changes $\left(r_{\text {spearman }}(27)=0.565 ; \mathrm{pFDR}=0.014\right)$ in the probiotics group only and independent of potential confounders (i.e., age, BMI, and baseline dietary fiber intake). That is subjects with a higher increase in Ruminococcaceae_UCG-003 abundance after probiotics were also more protected from negative effects of stress on working memory after probiotic supplementation. The bacterial taxa showing an increase in relative abundance in the probiotics group are plant fiber degrading bacteria and produce short-chain fatty acids that are known for their beneficial effect on gut and brain health, e.g., maintaining intestinal-barrier and blood-brain-barrier integrity. This study shows that gut microbial alterations, modulated through probiotics use, are related to improved cognitive performance in acute stress circumstances.
\end{abstract}

\footnotetext{
Correspondence: Mirjam Bloemendaal (mirjam.bloemendaal@radboudumc.nl) ${ }^{1}$ Departments of Psychiatry and Human Genetics, Radboud University Medical Center, Nijmegen, the Netherlands

${ }^{2}$ Laboratory of Microbiology, Wageningen University, Wageningen, The Netherlands

Full list of author information is available at the end of the article These authors contributed equally: Mirjam Bloemendaal, Joanna Szopinska-Tokov

These authors jointly supervised this work: Alejandro Arias Vasquez, Esther Aarts
}

\section{Introduction}

Stress, regardless of its origin (physical, mental, or social), activates the hypothalamic-pituitary-adrenal (HPA) axis ${ }^{1}$; a neuroendocrine system that controls the body's stress response. Stress-induced glucocorticoids released by the adrenal cortex impact many tissues in the body, including the brain where it affects cognitive performance (e.g., working memory ${ }^{2}$ ). Moreover, stress plays an important role in the neurobiology of mood disorders, including depressive and bipolar disorders ${ }^{3}$. The systemic

\section{(c) The Author(s) 2021}

(c) (i) Open Access This article is licensed under a Creative Commons Attribution 4.0 International License, which permits use, sharing, adaptation, distribution and reproduction c. in any medium or format, as long as you give appropriate credit to the original author(s) and the source, provide a link to the Creative Commons license, and indicate if changes were made. The images or other third party material in this article are included in the article's Creative Commons license, unless indicated otherwise in a credit line to the material. If material is not included in the article's Creative Commons license and your intended use is not permitted by statutory regulation or exceeds the permitted use, you will need to obtain permission directly from the copyright holder. To view a copy of this license, visit http://creativecommons.org/licenses/by/4.0/. 
effects of stress include mediation by the gut-brain axis (GBA) (for a review see Foster et al. ${ }^{4}$ ). This axis refers to the bidirectional communication between the gastrointestinal tract and the central nervous system, which is not only mediated by endocrine signaling including hormones and other neuro-active metabolites, but also by the vagus nerve and by the immune system ${ }^{5}$. The gut bacteria (gut microbiota) modify the functioning of the $\mathrm{GBA}^{6}$, making it a key player in behavior and stress reactivity ${ }^{4}$. A strong illustration of the role of the microbiota in the GBA and its effect on (healthy) behavior are the results of the experiments carried out in germ-free mice (i.e., mice reared in a germ-free environment preventing colonization of the intestinal tract) or animals subjected to wash out of their bacterial community by antibiotics. These animal models clearly show cognitive impairments, multiple behavioral disturbances such as altered anxiety responses, learning problems, and exaggerated stress reactivity $^{7,8}$. Sudo et al. ${ }^{9}$ observed altered HPA axis functioning in germ-free mice, i.e., amplified corticosterone response to stress induced by physical restraint, compared with control mice. The gut microbiota is a promising target for protection against negative effects of stress, as it is modifiable through e.g., diet or probiotics ${ }^{4}$. Probiotics are defined as "live, micro-organisms, which induces a health benefit to the host when administered in adequate amounts"10. Use of probiotics can be advised in case of irregularities in digestion and or stool composition such as diarrhea, aiming to re-establish more diverse gut microbiota abundance and reduce gastrointestinal complaints ${ }^{11}$. With the increasing awareness of the importance of the link between the microbiota-GBA in mood and stress-related symptoms, the potential of probiotics in protecting and or restoring these symptoms has been explored $^{12,13}$.

In the above-mentioned experiment in germ-free mice, Sudo et al. ${ }^{9}$ showed that the amplified corticosterone response to stress was normalized after supplementation with the bacterial strain Bifidobacterium infantis. Other animal studies showed that probiotics reduce anxious behaviors in response to physical stressors: i.e., less defensive probe burying after receiving shocks when supplemented with Lactobacillus helveticus R0052 and Bifdobacterium longum $R 0175$ for two-weeks ${ }^{14}$; more time spent in an open field after hypothermia when supplemented with L. rhamnosus for 29 days $^{15}$; and reduced immobilization time in a forced swim test after 5-week supplementation of the same multi-species probiotics mix as used in this study ${ }^{16}$. In human randomized placebocontrolled trials (RCTs), probiotics have also demonstrated beneficial effects on physical and/or psychological complaints in response to experimental acute stress paradigms or daily life stress. For example, subjects showed reduced depressive symptoms after 30-days of supplementation of the same 2-strain probiotic product effective in the animal study mentioned above ${ }^{14}$. Adults experiencing moderate stress symptoms on the Perceived Stress Scale (PSS-10) at baseline reported fewer of these symptoms after 8-weeks of use of Lactobaccilus Plantarum DR7 strain $^{17}$ or after 12-weeks of use of the P8 strain of the same species ${ }^{18}$. Reduced abdominal complaints during exam stress were observed using Lactobacillus casei for 8 weeks ${ }^{19}$. Similarly, eight-week supplementation of the Lactobacillus casei strains Shirota YIT 9029 relieved abdominal pain and exam stress ${ }^{20}$. After an acute stress paradigm, i.e., the socially evaluated cold pressor test (SECPT), fewer self-reported anxiety symptoms were observed after 4 weeks of Bifidobacterium longum 1714 use than after placebo ${ }^{21}$. In contrast, in two other RTCs in healthy volunteers, no alterations in stressrelated measures were observed after two weeks of supplementation of Lactobacilli, Bifidobacteria, and Streptococci strains $^{22}$ or after eight-week Lactobacillus rhamnosus supplementation ${ }^{23}$. Thus, although probiotics have been shown to affect human stress-related measures in some studies, the effects are not always replicated and more research is needed to understand whether and which gut microbial changes underly these cognitive effects.

The negative effects of (acute) stress on cognitive functioning have been rarely assessed in a probiotics trial. The double-blind RCT reporting fewer stress symptoms after 8-weeks Lactobaccilus Plantarum DR7 strain exposure also observed probiotic-induced improvements in emotion recognition speed and verbal memory speed ${ }^{17}$. Allen et al. observed fewer stress (SECPT) induced errors on a visuospatial memory task after 4 weeks of Bifidobacterium longum $1714^{21}$. Moreover, Papalini et al. ${ }^{24}$ assessed the effect of 4-week multi-strain probiotics supplementation on neurocognitive functioning in a double-blind RTC in healthy female volunteers. Probiotics protected against working memory detriments caused by an acute stress paradigm. That is, the probiotics group performed better after (versus before) the SECPT on a digit span backward test compared with the placebo group. Furthermore, this protecting effect of probiotics on the working-memory decline after stress was associated with changes in prefrontal cortex fMRI signal during cognitive control. From the above studies, it is, however, unclear how probiotics could exert their effect on central neural processing and cognitive performance. One pathway underlying the effects of probiotics on the GBA could be a specific alteration in microbial composition. Measuring probiotics-induced gut microbial alterations can help identify which bacteria mediate the protecting effects of probiotics on mental health and cognitive performance and can provide mechanistic insights into e.g., metabolite production and other microbial functions ${ }^{25}$. Yet, almost none of the above-mentioned studies report the effect of 
probiotics supplementation on gut microbial profiling. Kato-Kataoka et al. ${ }^{20}$ did observe higher alpha gut microbial diversity in the probiotics group in addition to lowered stress symptoms. However, the authors did not report an association between these measures of stress and microbiota. Another microbiota-GBA study assessed the effects of a Lactobacilli and Lactococci probiotics mixture on the gut microbial composition and emotional memory of healthy volunteers and found that increased memory was associated with decreased abundance of nine genera $^{26}$. However, these results were not observed in the context of stress-reactivity. Here, we aimed to assess how probiotics can buffer against the detrimental effects of stress on cognition by investigating the link with the probiotics-induced changes in the gut microbiota. For this, we used the data of the Papalini et al. ${ }^{24}$ study, where 28-days multi-species probiotics versus placebo supplementation were shown to improve working memory (i.e., digit span backward) performance after an acute stressor (i.e., the socially evaluated cold pressor test) in healthy female volunteers. We extend this work by assessing, first, whether this probiotics product alters gut microbial composition versus placebo and, second, whether this probiotics' effect on gut microbial composition relates to the probiotics' protective effect on stress-induced working memory changes.

\section{Methods}

\section{Subjects}

Healthy female subjects, aged between 18 and 40 years old were included (see Supplementary Materials for further inclusion criteria). A total of 61 healthy subjects were tested, of which one subject was excluded from the study due to high scores on the Beck Depression Inventory questionnaire (BDI), potentially indicating depression. For two subjects the pre-intervention fecal material was lacking, resulting in 58 subjects for the gut microbiota analysis $(n=27$ in the probiotics group, $n=31$ in the placebo group) (see Supplementary Fig. 1). Two other subjects scored poorly on the behavioral tasks and were excluded, resulting in a total sample-including both microbiota and neuro-cognitive data-of 56 subjects (probiotics group: $n=27$, mean age $=21.8, \mathrm{SEM}=0.5$, mean $\mathrm{BMI}=21.9, \mathrm{SEM}=0.32$; placebo group: $n=29$, mean age $=22.4, \mathrm{SEM}=0.53$, mean $\mathrm{BMI}=21.7, \mathrm{SEM}=$ 0.30 ). These exclusions although not pre-established were made to ensure a sample representative of the healthy population with reliable task performance. The groups did not differ in age, BMI, and neither in baseline dietary intake as reported in the Food Frequency Questionnaire Dutch Healthy Diet (FFQ-DHD) ${ }^{27}$, see Supplementary Table 1. The study was conducted following the Declaration of Helsinki with human subjects and the complete procedure was approved by the local Ethics Committee (CMO Arnhem-Nijmegen, NL55406.091.15) and registered at the Dutch trial register (protocol number: NTR5845). Written informed consent was obtained from each participant. The current analysis on gut microbial composition is an exploratory analysis for which no sample size was determined. The sample size is based on the sample needed for the effect of probiotics on neuro-cognitive measures as reported in seeing Papalini et al. $^{24}$ which was $n=30$ per intervention group. For an exhaustive description of the methods in this study, see Papalini et al. ${ }^{24}$, of which this study is an extension.

Procedure Subjects were tested on two days: the first day (baseline), before the intervention started, and the second day after 28 days (four weeks) of probiotics or placebo administration. The identical test sessions included cognitive testing in and outside the MRI scanner, an acute stress intervention, and fecal material collection before starting and after the last day of the intervention (see Fig. 1).

Relevant to the current analyses, the working memory task (digit span forward and backward) was performed before and after the SECPT. The SECPT is an established

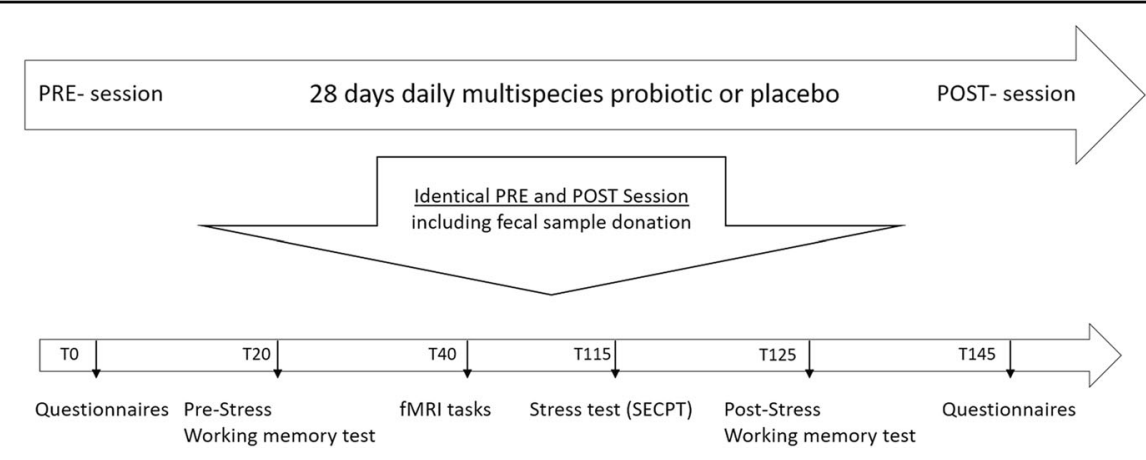

Fig. 1 Overview of the testing sessions. Each participant was tested twice, before and after 4 weeks of supplementation with probiotics/placebo. The procedure of the two sessions was the same (i.e., subjects performed the same tests in the same order). Tx: $x$ minutes since the start of the test session. SECPT: socially evaluated cold pressor test. 
stress-inducing paradigm consisting of both a physical and a social stressor ${ }^{28}$. Also in this experiment, the SECPT increased subjective feelings of stress measured with Visual Analogue Scales (VAS) and increased physiological measures, i.e., heart rate, blood pressure, and cortisol levels, in our subjects (see Fig. 4 in Papalini et al. ${ }^{24}$ ). During the digit span task, subjects listen to a series of numbers and are instructed to repeat each series correctly (digit span forward) or repeat it backward (digit span backward). Following a correct response, increasingly longer sequences are presented to the participant. Different series of numbers are used on each occasion (before and after stress on the first and second test day) to avoid long-term memory effects. In the $\mathrm{SECPT}^{28}$, subjects were instructed to hold their hand in a bucket of ice water $\left(0-3^{\circ} \mathrm{C}\right)$ for as long as possible (limited to $3 \mathrm{~min}$ ) under the surveillance of a video camera and an unfamiliar, disesteem expressing researcher. After the SECPT, the digit span was re-tested, thereby enabling assessment of stress on working memory performance. As in our previous work ${ }^{24}$, we only focus on the digit span backward, given that SECPT specifically influenced this type of working memory modulation instead of simply working memory maintenance that is needed in the digit span forward $^{29}$. Throughout the test session, VAS was used to assess well-being (5 times), heart rate and blood pressure were measured ( 7 times), and saliva samples (5 times) were obtained. Three fMRI paradigms measuring different aspects of emotion and cognition were performed while scanning. Once per test session, we further evaluated mood, emotional state, sensitivity, and diet. The total test session lasted almost $3 \mathrm{~h}$. The effects of the probiotics versus placebo intervention on these measures have already been reported in Papalini et al. ${ }^{24}$.

Intervention The used probiotics product Ecologic ${ }^{\circledR}$ Barrier (Winclove, The Netherlands) consists of the following bacterial strains: Bifidobacterium bifidum W23, Bifidobacterium lactis W51, Bifidobacterium lactis W52, Lactobacillus acidophilus W37, Lactobacillus brevis W63, Lactobacillus casei W56, Lactobacillus salivarius W24, Lactococcus lactis W19, and Lactococcus lactis W58. The total cell count was $2.5 \times 10^{9}$ colony forming units ( $\mathrm{cfu}$ ) per gram, and subjects consumed $2 \mathrm{~g}$ per day i.e., $5 \times 10^{9}$ cfu per day. More information on the product in the Supplementary Materials.

Microbiome sample processing Fecal sample collection Fecal samples were collected by the subjects at home using a validated protocol by OMNIgene.GUT kit (DNAGenotek, Ottawa, CA). The material was aliquoted into $1.5 \mathrm{ml}$ Eppendorf tubes and stored in $-80^{\circ} \mathrm{C}$ until further laboratory processing. Bacterial DNA isolation and sequencing For bacterial DNA extraction, $50 \mathrm{mg}$ of feces was separated from $200 \mu \mathrm{l}$ of OMNIgene.GUT kit buffer by centrifugation at $1400 \mathrm{rcf}$ at $16^{\circ} \mathrm{C}$ for $5 \mathrm{~min}$.
Microbial DNA was isolated from fecal pellets using the Maxwell ${ }^{\circledR} 16$ Instrument (Promega, Leiden, The Netherlands) as described previously ${ }^{30}$. DNA purification was performed with a customized kit (AS1220; Promega) using $250 \mu \mathrm{l}$ of the final supernatant pool. The V4 region of $16 \mathrm{~S}$ ribosomal RNA (rRNA) gene was amplified in duplicate PCR reactions for each sample in a total reaction volume of $50 \mu \mathrm{l}$. The $\mathrm{V} 4$ region was targeted by using previously reported primers for this region: $515 \mathrm{~F}\left(5^{\prime}\right.$ GTGYCAGCMGCCGCGGTAA)-806R (5' GGACTACN VGGGTWTCTAAT) ${ }^{30}$. We included a PCR negative sample to assess the contamination introduced during this step. The purified samples were used to prepare libraries for the Illumina HiSeq PE300 (paired-end, $300 \mathrm{bp}$ ) sequencing platform (GATC Biotech AG, Konstanz, Germany), with final loading concentrations of $200 \mathrm{ng} / \mu \mathrm{l}$. For more information on the wet lab procedure, see refs. ${ }^{31,32}$

\section{Data analyses bioinformatics}

Using the NG-Tax 16S rRNA pipeline ${ }^{33}$, taxonomic information was assigned. Briefly, the pipeline can be defined by three core elements: (i) barcode-primer filtering, (ii) operational taxonomic unit (OTU) picking, and (iii) taxonomic assignment using the SILVA reference database (version 128). Two filtering steps were applied to the output file (BIOM-file) of NG-Tax. First, a genus was filtered based on a $10 \%$ prevalence cutoff. Second, a sample with less than $10 \%$ of genera was removed (see ref. ${ }^{31}$ ). To avoid bias in assessing the effects of the intervention, filtering was done on the pre-intervention session. In total, 9,819,945 high-quality sequences were obtained from all samples after NG-Tax pipeline. These sequences were represented by 1043 OTUs and 175 genera. After the filtering steps, we obtained 9,681,326 sequences represented by 898 OTUs and 86 genera. No samples were removed during these steps.

\section{Statistical analyses}

The analyses followed a two-step approach (see Fig. 2). Step 1: assessing effects of probiotics on gut microbiota (community and composition analysis) and step 2: association between effects of probiotics on gut microbiota and neurocognitive measures. Step 1 served as a selection criterium for the association analysis in step 2. Overall, tests are performed two-sided and corrected for multiple comparisons. The biom file used for these analyses is available at $10.6084 / \mathrm{m} 9$.figshare.13614494. The raw sequences, QIIME, and R code used to analyze gut microbial composition are available upon request.

Community analyses Alpha-diversity: Alpha-diversity was estimated in three ways using QIIME 1.9.1 ${ }^{34}$ : (i) a richness measure counting observed OTUs, (ii) Shannon Index taking into account also abundance of the counted OTU's 


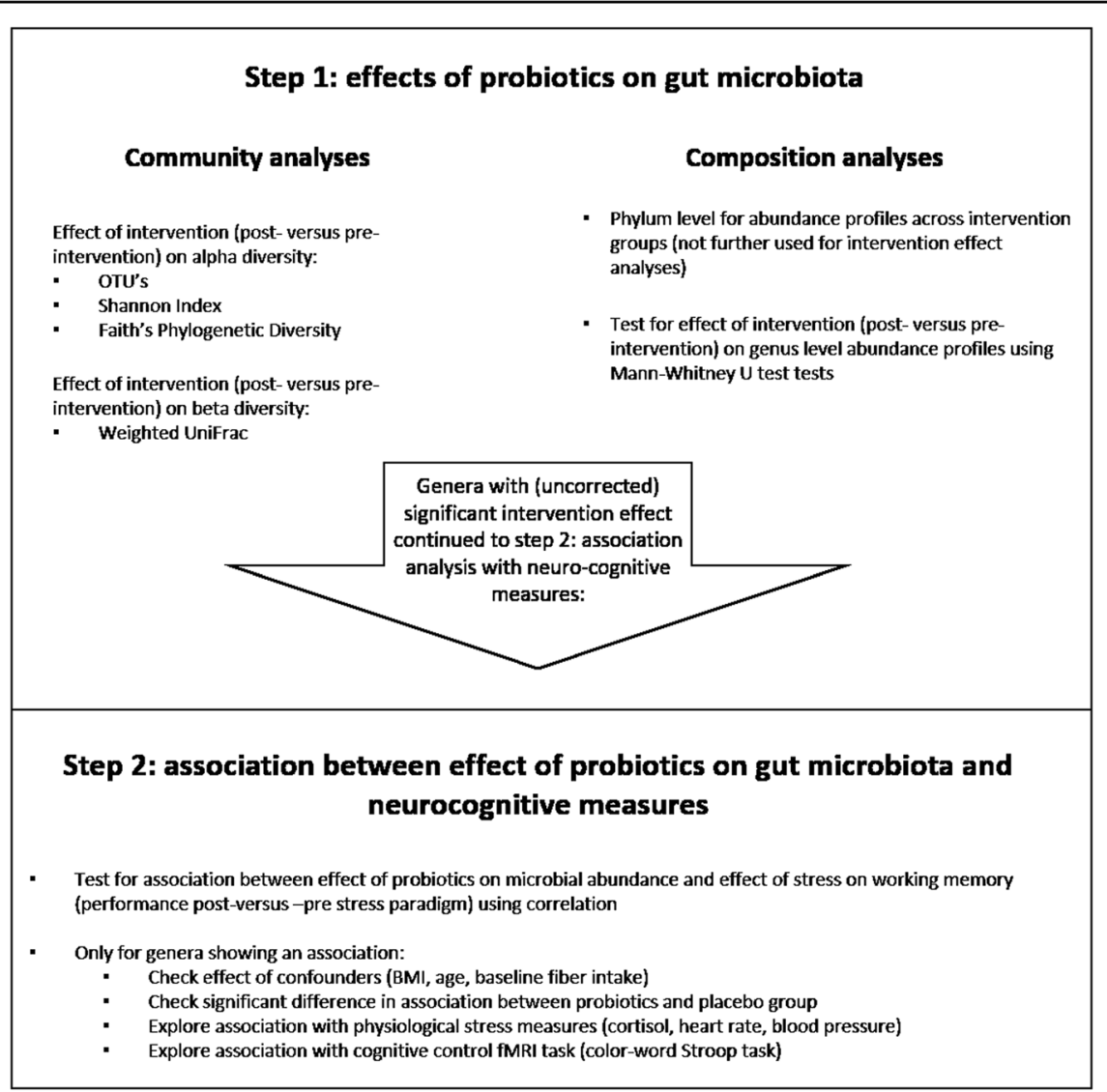

Fig. 2 Analysis flow chart. In a first step, community dynamics were compared between intervention (post-intervention versus pre-intervention) groups, and the composition of the two intervention groups was compared at the genus level. Secondly, the results of step 1 were used as a selection criterium to associate gut microbial candidates with neuro-cognitive measures.

and (iii) Faith's Phylogenetic Diversity, taking into account the evolutionary phylogenetic structure. After normality was verified, repeated measures ANOVAs were performed on between-subjects factor Intervention (Placebo, Probiotics) and within-subjects factor Time (Pre, Post), assessing Time (Pre, Post) * Intervention (Placebo, Probiotics), as well as a priori, hypothesized simple effects. The results are presented as differences (delta) between the pre-intervention and postintervention (after 4 weeks), symbolized by $\Delta$.

Beta-diversity: The beta-diversity was calculated based on weighted UniFrac ${ }^{35}$. Weighted UniFrac is a distance metric; explaining differences in the relative abundances between microbial communities based on their evolutionary phylogenetic structure. Permutation testing was performed in $\mathrm{R}$ (version 3.6.3) using the function vegan::adonis ${ }^{36}$, testing for interaction effects of Time (Pre, Post) * Intervention (Placebo, Probiotics), as well as a priori hypothesized simple effects in the weighted UniFrac distance metric, accounting for repeated measures by using the 'strata' argument when testing effects of Time. To visualize intervention effect on beta-diversity Constrained Analysis of Principal Coordinates (CAP) was performed using the $\mathrm{R}$ function vegan::capscale ${ }^{37}$.

\section{Composition analyses}

Taxonomic Differences: The differences in microbial communities between pre-intervention and postintervention groups were determined at the phylum and genus levels, based on the bacterial relative abundance profiles. Significant differences in relative abundance difference values, post-intervention versus pre-intervention (symbolized by $\Delta$ ), between probiotics and placebo groups were identified by non-parametric Mann-Whitney $U$ tests. Critically, this test on the genera altered by the probiotics intervention was used as a selection criterium for step 2: association analyses with neuro-cognitive measures, thereby restricting the number of tests for this second research question. Of these, genera with relative abundance levels differing between groups at baseline were not further analyzed (see Fig. 2).

Association analyses between intervention-induced changes in the gut microbiota and neuro-cognition: As reported in Papalini et al., after one-month supplementation of probiotics, the scores of the digit span backward improved after the stress-inducing SECPT paradigm, while this effect was not observed in the 
placebo group. The association analysis focused primarily on this result: assessing whether the protective effect of probiotics on stress-induced working memory changes is explained by changes in gut microbial composition due to probiotics use. To this aim, firstly, only genera altered due to probiotics exposure (i.e., post-pre increase or decrease $(\Delta)$ in relative abundance after probiotics versus placebo) were selected for the downstream analysis.

Using correlation analyses, these genera were screened for a relation with the effect of probiotics (post-pre) on working memory (digit span backward, DS) after versus before stress, i.e., (DSafter-DSbefore stress) ${ }^{\text {post-intervention }}$ (DSafter-DSbefore stress) ${ }^{\text {pre-intervention }}$ (see Fig. 2).

Due to the overall skewness of the microbiota data, twotailed non-parametric Spearman correlations were performed. Only results passing False Discovery Rate (FDR) correction were analyzed in a multiple regression analysis. Here, the effect of potential confounders was assessed by adding BMI, age, and baseline fiber intake (measured by the DHD) in a linear regression model. The efficacy of probiotics may vary with age and BMI due to metabolic and gastro-intestinal differences ${ }^{38,39}$. Although no changes in the dietary patterns were observed over the course of the intervention, baseline fiber intake may still be a modulator of the effect of probiotics as these serve as a nutrient source for the microbiota ${ }^{40}$.

The specificity of the association between the effect of probiotics on gut microbial abundance and stress-related working memory was assessed by comparing the regression slopes between the probiotics and placebo groups. This was done by creating a dummy variable that divided the intervention groups and subsequently creating an interaction term between this dummy variable and the genus variable. This interaction term was then added in a new regression, assessing whether the relative abundance of the selected genus differentially affects stress-induced working memory changes in the two intervention groups. For results on the secondary analyses that were performed on fMRI signal and physiological stress measures, see Supplementary Methods. The statistical analyses were performed using IBM SPSS statistics (package version 23).

\section{Results}

\section{Gut microbiota composition before intervention}

The intestinal microbiota of the 58 subjects before the intervention (Pre) was typical for healthy individuals; dominated largely by the phyla Firmicutes $(68.0 \%)$ and Bacteroidetes (19.5\%), accounting for up to $87.5 \%$ of the intestinal microbial communities. Other observed phyla were Actinobacteria (8.7\%), Proteobacteria (1.5\%), Verrucomicrobia $(1.4 \%)$, Euryarchaeota $(0.4 \%)$, Tenericutes $(0.29 \%)$, and Cyanobacteria $(0.25 \%)$.

\section{Effect of probiotics on gut microbiota community}

The three alpha diversity measures did not show significant interaction effects between Time and Intervention, or simple effects in the probiotics and placebo groups, all $p>0.05$ (Supplementary Fig. 2). Beta-diversity at the operational taxonomic unit (OTU) level did not show a significant interaction between Time (pre, post) and Intervention (placebo, probiotics) $\left(p=0.244, r^{2}=\right.$ 0.003). However, there was a main effect of Time across Interventions $\left(p=0.011, r^{2}=0.01\right)$. That is, the postintervention samples were more similar among each other compared with the pre-intervention samples. Constrained analysis of principle coordinates (CAP) ordination method was used to visualize the main effect of Time (accounting for $R^{2}=1 \%$ variability in the dataset) (Fig. 3). Exploratory post-hoc analyses suggest that the Time effect is more likely driven by changes within the probiotics group (post versus pre-4-week of intervention $(p=0.008)$, rather than the placebo group $(p=0.21)$ (Supplementary Table 2). It's critical to note that the absence of Time by Intervention interaction indicates that that probiotic and placebo intervention affected the beta diversity in a similar fashion as shown in Fig. 3.

Effect of probiotics on gut microbiota composition: At the phylum level, we did not find significant differences between a post-pre increase or decrease $(\Delta)$ in relative abundance after probiotics versus placebo; Supplementary Table 3). At the genus level, we identified nine genera with FDR-uncorrected significant changes in relative abundance in the probiotics group (post-intervention versus pre-intervention). One of these nine (Parabacteroides) was different at baseline between the probiotics and placebo group and was therefore not taken into consideration for further analyses (Table 1, Supplementary Fig. 3A-H). Another classification (Lachnospiraceae_g_) is in fact a sequence for which no classification

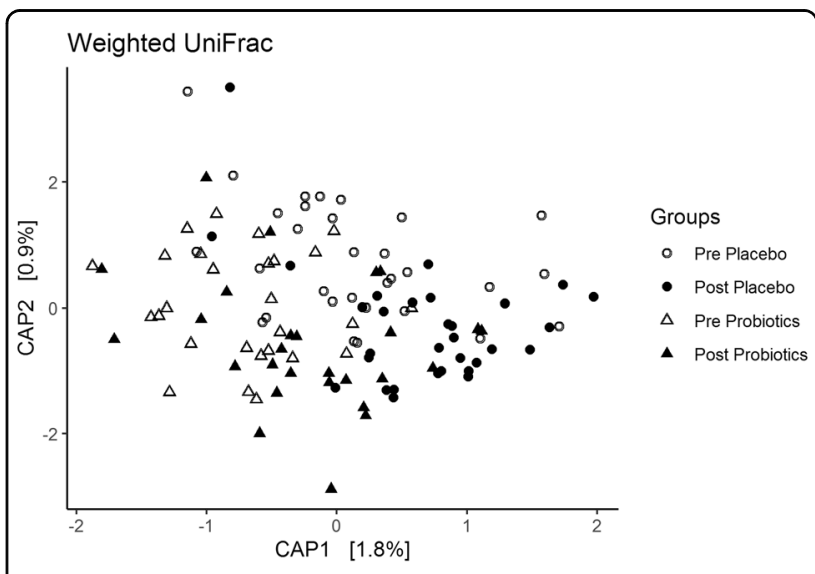

Fig. 3 Beta diversity. Weighted Unifrac dissimilarity matrix plotted using Canonical Analysis of Principal Coordinates (CAP) ordination for all groups and timepoints. 
Table 1 Comparison of genus relative abundance post-pre intervention changes $(\Delta)$ between probiotics and placebo groups.

\begin{tabular}{|c|c|c|c|c|c|}
\hline & $\begin{array}{l}\Delta \text { Probiotics } \\
\text { mean rank }\end{array}$ & $\Delta$ Probiotics mean (range) $^{a}$ & $\begin{array}{l}\Delta \text { Placebo } \\
\text { mean rank }\end{array}$ & $\Delta$ Placebo mean (range) ${ }^{\mathrm{a}}$ & $P$-value \\
\hline Butyricimonas & 33.78 & $0.04(-0.26-0.45)$ & 25.77 & $-0.02(-0.29-0.01)$ & 0.014 \\
\hline Parabacteroides $^{c}$ & 34.78 & $-0.003(-0.83-0.69)$ & 24.90 & $-0.22(-1.87-2.51)$ & 0.026 \\
\hline Alistipes & 34.20 & $-0.05(-4.32-1.82)$ & 25.40 & $-0.18(-3.06-3.25)$ & 0.048 \\
\hline Christensenellaceae_R-7_group & 36.11 & $0.5(-1.34-3.14)$ & 23.74 & $-0.72(-8.10-3.33)$ & 0.005 \\
\hline Family_XIII_AD3011_group & 34.11 & $0.09(0.00-0.54)$ & 25.48 & $0.03(-0.14-0.52)$ & 0.032 \\
\hline f_Lachnospiraceae;g_ & 23.70 & $-1.09(-10.67-10.93)$ & 34.55 & $1.17(-9.95-9.21)$ & 0.015 \\
\hline Ruminococcaceae_UCG-003 & 34.30 & $0.07(-0.27-0.52)$ & 25.32 & $-0.04(-0.68-0.34)$ & 0.038 \\
\hline Ruminococcaceae_UCG-005 & 34.70 & $0.29(-1.42-1.82)$ & 24.97 & $-0.27(-5.69-1.41)$ & 0.028 \\
\hline Ruminococcaceae_UCG-010 & 35.04 & $0.09(-0.29-0.95)$ & 24.68 & $-0.06(-0.83-0.98)$ & 0.010 \\
\hline
\end{tabular}

${ }^{a}$ Mean and range of the difference scores are given to provide descriptive statistics; these values have no relation with the $p$-values.

${ }^{\mathrm{b}} P$-values (uncorrected) are given for non-parametric Mann-Whitney $U$ test assessing the differences in rank order. These non-parametric rank tests were performed due to the overall skewness of the microbiota data including many zero values. A cut-off was placed including maximally $90 \%$ zero values (see the "Methods" section for more details).

'Different at baseline.

to an OTU could be made with sufficient confidence, resulting in a grouping of OTU's with insufficient confidence. This grouping of genera within the Lachnospiraceae family was also not taken forward for further analysis.

Association between gut microbiota composition and neurocognitive measures: Seven genera were used to assess a correlation with the protective effect of probiotics on working memory (digit span backward scores) after stress ${ }^{24}$. Of these, the post-change versus pre-change in the relative abundance of genus Ruminococcaceae_UCG-003 was significantly correlated with the change in the effect of stress on working memory in the probiotics group $\left(r_{\text {spearman }}(27)=0.565 ; \mathrm{pFDR}=0.014\right)$ (see Fig. 4). In the probiotics group, a larger increase (post-intervention versus pre-intervention) in the relative abundance of Ruminococcaceae_UCG-003 was associated with higher (post-intervention versus pre-intervention) stress-related working memory performance (digit span backward, post-SECPT versus pre-SECPT). Linear regression was subsequently used to assess potential confounders in the relationship between probiotics-induced changes in working memory performance after stress and Ruminococcaceae_UCG-003 ( $\Delta$ scores). Age, BMI at baseline, as well as baseline dietary fiber intake were identified as potentially modulating the effect of probiotics and its relation with neurocognitive measures. None of these confounders had a significant effect in the model (model without potential confounders; Adjusted $R^{2}=0.14$, Standardized Beta Ruminococcaceae_UCG-003 $=0.45$, $p=0.018$, a model with potential confounders; Standardized Beta Ruminococcaceae_UCG-003 $=0.50, p=0.022$, potential confounders: Age: Standardized Beta $=-0.117$, $p=0.565$, BMI: Standardized Beta $=-0.133, p=0.661$, baseline reported fiber intake: Standardized Beta $=0.170$, $p=0.814$ ). Dietary intake (DHD-FFQ) scores were not affected by the intervention; all $p>0.05$ (for scores see Supplementary Table 1). The association between probiotics-induced changes in working memory performance after stress and Ruminococcaceae_UCG-003 ( $\Delta$ scores) was not significant in the placebo group $(B=-0.179, p=0.296)$. Indeed, the association was significantly greater for the probiotics' than for the placebo group: the regression coefficients for the effect of Ruminococcaceae_UCG-003 on stress-induced working memory changes in the probiotics and placebo groups were significantly different (Slope comparison interaction term: Beta $=-0.614, p=0.009$ ).

For reference, plots on the non-significant relation between the other seven genera and digit span scores are provided in Supplementary Fig. 4. The secondary analysis on the neural signal during cognitive control and physiological measures did not give significant associations with change in Ruminococcaceae_UCG-003 abundance, see Supplementary Results.

\section{Discussion}

Here, we show that the effects of multi-strain probiotics on stress-related cognitive performance are associated with changes in the gut microbiota composition. This association was exclusive to the probiotics group and independent of the potential confounders' age, BMI, and baseline dietary fiber intake. 


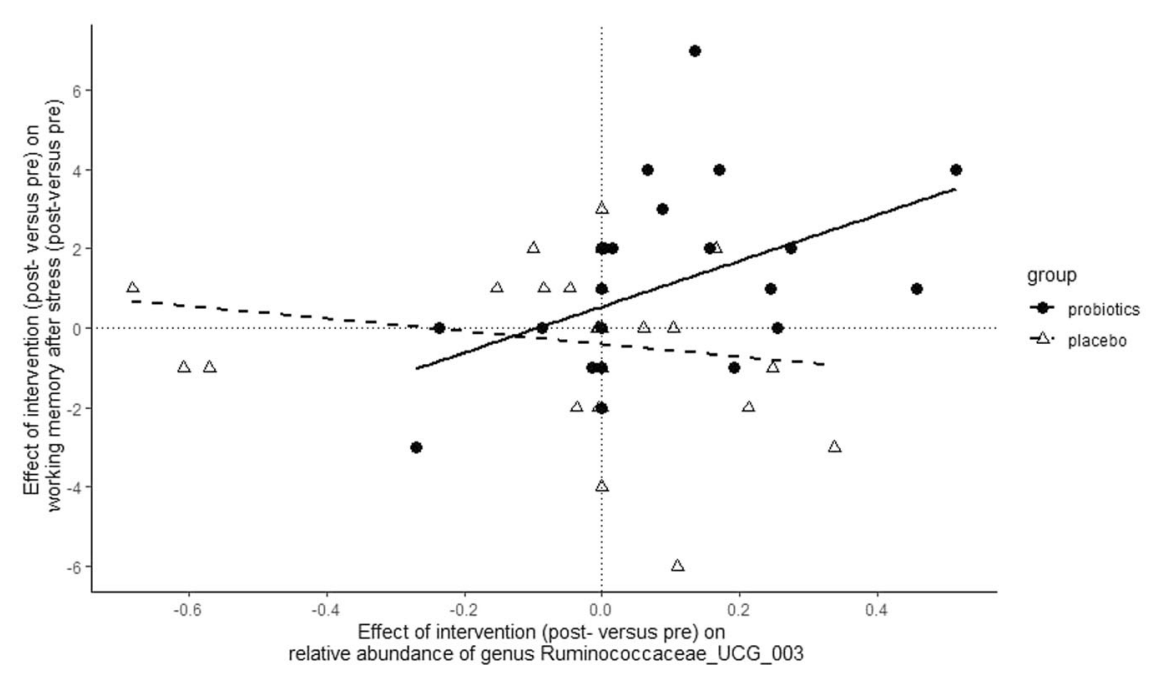

Fig. 4 Association between the intervention-induced change in the relative abundance of genus Ruminococcaceae_UCG-003 and intervention-induced protection of stress-induced working memory changes. Subjects with a higher increase in Ruminococcaceae_UCG-003 abundance after probiotics (right side of the $x$-axis) were also more protected from negative effects of stress on working memory after probiotics (upper side of the $y$-axis).

Healthy, female subjects took multi-strain probiotics or placebo for 28 days and donated fecal material at baseline and after the intervention period. In an identical preintervention and post-intervention session, subjects performed a working memory task before and after an acute stress manipulation. Gut microbial beta diversity did not show a significant interaction between Intervention and Time but changed over time across intervention groups. In terms of gut microbial composition, we observed increased relative abundance of the genera Butyricimonas, Alistipes, Christensenellaceae_R-7_group, Family_XIII_AD3011_group, Ruminococcaceae_UCG-003, Ruminococcaceae_UCG-005, and Ruminococcaceae_UCG-010 in the probiotics versus the placebo group, (uncorrected for multiple comparisons). These genera showing an uncorrected higher relative abundance are part of known abundant taxa observed in the healthy human gut $^{41,42}$. Of these genera selected for a second analysis step, only Ruminococcaceae_UCG-003 was significantly associated with the effect of probiotics on working memory performance after stress, where subjects with a larger probiotics-induced increase in Ruminococcaceae_UCG-003 abundance also showed a greater protective effect of probiotics on stress-induced working memory changes.

It is the first time that the effects of these multi-strain probiotics on gut microbiota composition are tested in humans. Our results show uncorrected probiotics-induced changes in the relative abundance of several genera. These are associated with gastrointestinal health and lifestyle choices; Ruminococcaceae_UCG-003, and uncorrected also Butyricimonas, Family_XIII_AD3011_group, f_Lachnospiraceae, were found to be less abundant in healthy men reporting to smoke and drink alcohol ${ }^{43}$. Genus Alistipes, Ruminococcaceae_UCG-005, and Christensenellaceae R-7 group have been previously associated with a healthy gut microbial composition when compared with subjects with gastro-intestinal diseases ${ }^{44}$. Though not all of these genera are classified in function, these four genera, as well as Butyricimonas, are known to plant fiber degraders and produce short-chain fatty acid (SCFAs) ${ }^{45-48}$. SCFAs perform a pivotal role in the $\mathrm{GBA}^{49}$, besides being a nutrient source for resident bacteria ${ }^{50}$. SCFAs beneficially affect the immune system, e.g., by the secretion of cytokines and $\mathrm{T}$ cell differentiation. Moreover, they protect intestinally and blood-brain barrier permeability and modulate the HPA axis through the vagal nerve ${ }^{50-54}$. Increased SCFA production may hence contribute to the protecting effect of probiotics on gut and brain health. While we find an association between stress-related cognitive performance and gut microbial abundance, none of the physiological stress measures associated with the gut microbial changes, which is in line with our previous findings that these stress measures were not affected by probiotics across the group ${ }^{24}$. The stress paradigm did activate the HPA axis as measured by blood pressure, heart rate, and cortisol ${ }^{24}$. Dampened cortisol responses after probiotics' use is not consistently observed in human RCTs: while Messaoudi et al. ${ }^{14}$ found lowered urinary levels of cortisol, Takada et al. ${ }^{19}$ only found lowered levels when pooling salivary cortisol measures from three trials, and Mohammadi et al. ${ }^{55}$ did not observe serum cortisol changes due to probiotics even though well-being was improved. The absence of physiological effects of the probiotics in the current trial suggests there are other 
pathways involved rather than HPA (de-)activation. For instance, the probiotics' intervention could have resulted in the prevention of stress-induced immune activation that is known to affect brain neurotransmitters, e.g., catecholamines, underlying cognitive performance ${ }^{56}$. In favor of such a potential pathway are results using the current multi-species probiotics mixture in rats, where the gut metabolite indolepropionic acid was increased ${ }^{16}$. Indolepropionic acid is known to improve intestinal barrier function and to limit neuro-inflammation ${ }^{57}$. Moreover, in the paper by Lin et al. Ruminococcaceae_UCG-003 abundance associated negatively with the inflammatory biomarker C-reactive protein ${ }^{43}$. SCFAs can also contribute to the reduction of stress-induced inflammation ${ }^{58,59}$. As we did not directly measure gut metabolites in stool or serum, nor inflammatory cytokines, we suggest future studies may look into these functional pathways of how probiotics support cognitive resilience to stress. We were not able to determine if the bacterial strains included in the "Ecologic ${ }^{\circledR B a r r i e r}{ }^{\prime}$ probiotic product changed after our intervention. The $16 \mathrm{~S}$ rRNA sequencing technique allows robust and consistent identification of taxa up to the genus category but not lower taxa (i.e., species). None of the genera that were increased by the intervention include any of the supplemented strains. When revising the genera including the strains present in the probiotics mixture, we observed a non-significant increase in relative abundance: genus Bifidobactium increased 1,1\% post-probiotics versus pre-probiotics, and genus Lactobacillus increased $0.5 \%$. The genus Lactococcus was not detected in the sample. It is important to note that the efficacy of probiotics does not depend on and is not expected to be limited to the colonization of the supplemented strains themselves. Probiotics can alter the relative abundance of multiple bacterial groups, but also affect community dynamics in several ways, e.g., through changes in $\mathrm{pH}$, by outcompeting bacteria by utilizing nutrients, by promoting the growth of resident bacteria through nutrient production, or by occupying adherence sites on the intestinal wall ${ }^{50}$. In fact, changes in gut microbial composition are not consistently found following probiotics' use ${ }^{50}$. Our findings of probiotic-induced changes in other genera than supplemented, therefore, contribute to the understanding of probiotics' supplementation in healthy human subjects. Our current findings are in need of replication, ideally in a larger sample and extending the findings to a male sample. Moreover, FFQ data as used in this study, only roughly matches fiber intake, with a slight underestimation as calculated in some studies ${ }^{60,61}$. The currently used short FFQ (15 min) was selected to control for large differences in adherence to the Dutch healthy diet guidelines and to obtain an estimate of fiber intake in order to control for this potential confounder. The FFQ values should in no way be interpreted quantitatively. Future studies that are interested in actual interactions between diet and probiotics supplementation should go for more extensive measures like $24 \mathrm{~h}$ recalls or food diaries. Nevertheless, another study similarly found variation in abundance of a Ruminococcaceae genus to be associated with neurocognitive measures: Bagga et al. ${ }^{62}$ tested, without a stress paradigm, the neuro-cognitive effects of similar probiotics product in healthy volunteers. They observed that irrespective of intervention, the OTU Ruminococcaceae_UCG_002 was negatively correlated with depressive feelings. Even though this finding does not involve Ruminococcaceae_UCG-003 specifically, the mentioned genus belongs to the Ruminococcaceae family, meaning they are evolutionary similar. With research into the microbiota-GBA accumulating, it is interesting to see whether the beneficial role of Ruminococcaceae genera in neuro-cognitive performance in health and disease is confirmed. Similar to our results, Bagga et al. ${ }^{62}$ also found that memory performance was improved by the probiotics supplementation, which was in their study associated with changes in the Bacteroides genera. This difference in the association between the studies, i.e., the currently observed Ruminococcaceae_UCG-003 versus Bacteriodes, might not be surprising given the different type of memory (working memory versus emotional memory), different type of probiotic product (Ecologic ${ }^{\circledR B}$ Barrier versus Ecologic ${ }^{8} 825$ ), and different sample (larger $n=56$ instead of $n=23$ for microbiota analyses] and more homogenous [only female] in our study). Another study investigated the effect of probiotics in exam-related stress in healthy adults (students) $^{20}$. In this study, they used a single strain (Lactobacillus casei strain Shirota YIT 9029, $1.0 \times 10^{11}$ CFU per $100-\mathrm{ml}$ bottle, 8 weeks supplementation; not overlapping with any in this study). The authors found the preservation of within-group diversity after probiotics compared with placebo, whereas in our study betweengroup diversity shifted in the probiotics group, and increased abundance of several genera was observed (on an uncorrected threshold). This difference in functional effects likely partly arises due to the different primer regions of the $16 \mathrm{~S}$ rRNA gene studied; V1-2 in ${ }^{20}$ and V4 in our study. These differences in study design (e.g., duration, dose), probiotics product used (e.g., single-strain or multistrain), gut microbiome analysis techniques (e.g., in $16 \mathrm{~S}$ rRNA primer amplicon, DNA extraction method) hamper comparability between probiotics' studies. Formal replication studies are needed to overcome this limitation to the probiotics field. Accumulation of probiotic trials in the microbiota-GBA field will increase knowledge on the effects, potentially by backtracking shared functional effects (on gut microbial composition, physiology, or behavior) to shared properties across these strains. Lastly, significant effects of probiotics on the global beta diversity measure were not observed in the current dataset. 
Variation in the gut microbial composition of healthy adults is a natural biological phenomenon, as well as technical variations e.g., due to sampling ${ }^{63}$. Interaction effects can only appear when an intervention effect is strong enough to supersede the natural temporal fluctuations in the community, which, given our results, seems not to be the case for the current probiotics intervention in this healthy adult sample. It is important to be aware that the community analyses, in this case, beta diversity, are not a pre-requisite for assessing changes in composition at (genus) taxonomy level ${ }^{64}$. They are separate approaches each assessing different aspects of the microbiome composition: beta diversity values are extracted from the distribution of OTU's, which is a different distribution than the taxonomic data. While it is of course true that the taxonomic characterization depends on the OTU distribution, taxonomic groups at the genus level are assigned based on sequence similarity, creating a different metric. The lack of global differences such as beta diversity does not preclude individual taxonomic differences at the genus level. In fact, relevant and significant intervention effects at the taxonomic level may drown in community analysis due to the nature of this global measure: a global tendency across all taxa.

In conclusion, 4-weeks supplementation of probiotics did not significantly increase the relative abundance of the gut microbiota at genus level versus placebo. However, a group of eight genera showed an increase (uncorrected) after probiotics compared to placebo. The increased relative abundance of the gut bacterial genus Ruminococcaccae_UCG-003 correlated significantly with the positive effects of probiotics on stress-induced working memory changes. In addition to our previous findings of probiotics supporting cognitive performance under stress relative to placebo ${ }^{24}$, our current exploratory results suggest that these beneficial effects may be related to changes in the gut microbiota community. More research into the functional effects of gut microbial changes would add to the understanding of probiotics' modulation of the gut-brain axis.

\footnotetext{
Author details

'Departments of Psychiatry and Human Genetics, Radboud University Medical Center, Nijmegen, the Netherlands. 'Laboratory of Microbiology, Wageningen University, Wageningen, The Netherlands. ${ }^{3}$ Donders Institute for Brain Cognition and Behaviour, Centre for Cognitive Neuroimaging, Radboud University, Nijmegen, the Netherlands. ${ }^{4}$ Laboratory for Biological Psychology, Katholieke Universiteit Leuven, Leuven, Belgium. ${ }^{5}$ Winclove Probiotics, Amsterdam, the Netherlands

Conflict of interest

The study was supported by the Dutch Ministry of Economic Affairs under the TKI Life Science and Health, project LSHM15034, and by Winclove Probiotics B.V., The Netherlands. Saskia van Hemert is employed at Winclove Probiotics B.V. This does not alter this authors' adherence to all publication policies on sharing data and materials. The remaining authors declare no competing interests.
}

\section{Publisher's note}

Springer Nature remains neutral with regard to jurisdictional claims in published maps and institutional affiliations.

Supplementary information The online version contains supplementary material available at https://doi.org/10.1038/s41398-021-01404-9.

Received: 30 June 2020 Revised: 24 March 2021 Accepted: 21 April 2021 Published online: 20 May 2021

\section{References}

1. Nicolaides, N. C. et al. Stress, the stress system and the role of glucocorticoids. Neuroimmunomodulation 22, 6-19 (2014).

2. Girotti, M. et al. Prefrontal cortex executive processes affected by stress in health and disease. Prog. Neuro-Psychopharmacol. Biol. Psychiatry 85, 161-179 (2018).

3. Turner, A. I. et al. Psychological stress reactivity and future health and disease outcomes: a systematic review of prospective evidence. Psychoneuroendocrinology 114, 104599 (2020).

4. Foster, J. A., Rinaman, L. \& Cryan, J. F. Stress and the gut-brain axis: regulation by the microbiome. Neurobiol. Stress 7, 124-136 (2017).

5. de Weerth, C. Do bacteria shape our development? Crosstalk between intestinal microbiota and HPA axis. Neurosci. Biobehav. Rev. 83, 458-471 (2017).

6. Cryan, J. F. et al. The microbiota-gut-brain axis. Physiol. Rev. 99, 1877-2013 (2019).

7. Luczynski, P. et al. Growing up in a bubble: Using germ-free animals to assess the influence of the gut microbiota on brain and behavior. Int. J. Neuropsychopharmacol. 19, 1-17. (2016).

8. Fröhlich, E. E. et al. Cognitive impairment by antibiotic-induced gut dysbiosis: analysis of gut microbiota-brain communication. Brain Behav. Immun. 56, 140-155 (2016).

9. Sudo, N. et al. Postnatal microbial colonization programs the hypothalamicpituitary-adrenal system for stress response in mice. J. Physiol. 1, 263-275 (2004).

10. Hill C et al. The International Scientific Association for Probiotics and Prebiotics consensus statement on the scope and appropriate use of the term probiotic. Nat. Rev. Gastroenterol. Hepatol. 11, https://doi.org/10.1038/nrgastro.2014.66 (2015).

11. Islam, S. U. Clinical uses of probiotics. Medicine 95, e2658 (2016).

12. Dinan, T. G., Stanton, C. \& Cryan, J. F. Psychobiotics: a novel class of psychotropic. Biol. Psychiatry 74, 720-726 (2013).

13. Sarkar, A. et al. Psychobiotics and the manipulation of bacteria-gut-brain signals. Trends Neurosci. 39, 763-781 (2016).

14. Messaoudi, M. et al. Assessment of psychotropic-like properties of a probiotic formulation (Lactobacillus helveticus R0052 and Bifidobacterium longum R0175) in rats and human subjects. Br. J. Nutr. 105, 755-764 (2011).

15. Bravo, J. A. et al. Ingestion of Lactobacillus strain regulates emotional behavior and central GABA receptor expression in a mouse via the vagus nerve. Proc. Natl Acad. Sci. USA 108, 16050-5 (2011).

16. Abildgaard, A., Elfving, B., Hokland, M. \& Wegener, G. Probiotic treatment reduces depressive-like behaviour in rats independently of diet. Psychoneuroendocrinology 79, 40-48 (2017).

17. Chong, H. X. et al. Lactobacillus plantarum DR7 alleviates stress and anxiety in adults: a randomised, double-blind, placebo-controlled study. Benef. Microbes 10, 355-373 (2019).

18. Lew, L. C. et al. Probiotic Lactobacillus plantarum P8 alleviated stress and anxiety while enhancing memory and cognition in stressed adults: a randomised, double-blind, placebo-controlled study. Clin. Nutr. 38, 2053-2064 (2019).

19. Takada, M. et al. Probiotic Lactobacillus casei strain Shirota relieves stressassociated symptoms by modulating the gut-brain interaction in human and animal models. Neurogastroenterol. Motil. 28, 1027-1036 (2016).

20. Kato-Kataoka, A. et al. Fermented milk containing Lactobacillus casei strain shirota preserves the diversity of the gut microbiota and relieves abdominal. Appl. Environ. Microbiol. 82, 3649-3658 (2016).

21. Allen, A. P. et al. Bifidobacterium longum 1714 as a translational psychobiotic: modulation of stress, electrophysiology and neurocognition in healthy volunteers. Transl. Psychiatry 6, https://doi.org/10.1038/tp.2016.191 (2016). 
22. Möller, C. M. et al. Influence of acute multispecies and multistrain probiotic supplementation on cardiovascular function and reactivity to psychological stress in young adults: a double-blind, randomized, placebo-controlled trial. Psychosom. Med. 79, 914-919 (2017).

23. Kelly, J. R. et al. Lost in translation? The potential psychobiotic Lactobacillus rhamnosus (JB-1) fails to modulate stress or cognitive performance in healthy male subjects. Brain Behav. Immun. 61, 50-59 (2017).

24. Papalini, S. et al. Stress matters: a double-blind, randomized controlled trial on the effects of a multispecies probiotic on neurocognition. Neurobiol. Stress 10 , 263673 (2018).

25. Suez, J., Zmora, N., Segal, E. \& Elinav, E. The pros, cons, and many unknowns of probiotics. Nat. Med. 25, 716-729 (2019).

26. Bagga, D., Louise, J., Koschutnig, K. \& Aigner, C. S. Probiotics drive gut microbiome triggering emotional brain signatures. Gut Microbes 9, 486-496 (2018).

27. van Lee, L. et al. The Dutch Healthy Diet index as assessed by $24 \mathrm{~h}$ recalls and FFQ: associations with biomarkers from a cross-sectional study. J. Nutr. Sci. https://doi.org/10.1017/jns.2013.28 (2013).

28. Schwabe, L, Haddad, L. \& Schachinger, H. HPA axis activation by a socially evaluated cold-pressor test. Psychoneuroendocrinology 33, 890-895 (2008).

29. Schoofs, D., Wolf, O. T. \& Smeets, T. Cold pressor stress impairs performance on working memory tasks requiring executive functions in healthy young men. Behav. Neurosci. 123, 1066-1075 (2009).

30. Fernández-Calleja, J. M. S. et al. Non-invasive continuous real-time in vivo analysis of microbial hydrogen production shows adaptation to fermentable carbohydrates in mice. Sci. Rep. 8, 1-16. (2018).

31. Szopinska-Tokov, J. et al. Investigating the gut microbiota composition of individuals with attention-deficit/hyperactivity disorder and association with symptoms. Microorganisms 8, https://doi.org/10.3390/microorganisms8030406 (2020).

32. Szopinska, J. W. et al. Reliability of a participant-friendly fecal collection method for microbiome analyses: a step towards large sample size investigation. BMC Microiol. 18, 1-11 (2018).

33. Ramiro-Garcia, J. et al. NG-Tax, a highly accurate and validated pipeline for analysis of $16 \mathrm{~S}$ rRNA amplicons from complex biomes. F1000Research 5, 1791 (2016).

34. Caporaso, J. G. et al. QIIME allows analysis of high-throughput community sequencing data. Nat. Methods 7, 335-336 (2011).

35. Lozupone, C. A., Hamady, M., Kelley, S. T. \& Knight, R. Quantitative and qualitative $\beta$ diversity measures lead to different insights into factors that structure microbial communities. Appl. Environ. Microbiol. 73 1576-1585 (2007).

36. Oksanen, A. J. et al. Package ' vegan'. (2019).

37. Morelan, I. A. et al. Microbiome variation in an intertidal sea anemone across latitudes and symbiotic states. 6, 1-13 (2019).

38. Arrieta, M.-C. et al. The intestinal microbiome in early life: health and disease. Front. Immunol. 5, 1-18. (2014).

39. Heintz, C. \& Mair, W. You are what you host: microbiome modulation of the aging process. Cell 156, 408-411 (2014).

40. Do Espírito Santo, A. P. et al. Fibers from fruit by-products enhance probiotic viability and fatty acid profile and increase CLA content in yoghurts. Int. J. Food Microbiol. 154, 135-144 (2012).

41. Tap, J. et al. Towards the human intestinal microbiota phylogenetic core. Environ. Microbiol. 11, 2574-2584 (2009).

42. Rajilić-Stojanović, M. \& de Vos, W. M. The first 1000 cultured species of the human gastrointestinal microbiota. FEMS Microbiol. Rev. 38, 996-1047 (2014).

43. Lin, R. et al. The effects of cigarettes and alcohol on intestinal microbiota in healthy men. J. Microbiol. 58, 926-937 (2020).

44. Mancabelli, L. et al. Identification of universal gut microbial biomarkers of common human intestinal diseases by meta-analysis. FEMS Microbiol. Ecol. 93 1-10. (2017).
45. Biddle, A., Stewart, L., Blanchard, J. \& Leschine, S. Untangling the genetic basis of fibrolytic specialization by lachnospiraceae and ruminococcaceae in diverse gut communities. Diversity 5, 627-640 (2013).

46. Flint $\mathrm{HJ}$ et al. Microbial degradation of complex carbohydrates in the gut Microbial degradation of complex carbohydrates in the gut @ 2012 Landes Bioscience. Do not distribute. 0976, https://doi.org/10.4161/gmic.19897 (2012).

47. Esquivel-Elizondo, S., Ilhan, Z. E., Garcia-Peña, E. I., Krajmalnik-Brown, R. Insights into butyrate production in a controlled fermentation system via gene predictions. mSystems 2, https://doi.org/10.1128/msystems.00051-17 (2017)

48. Vital, M., Karch, A. \& Pieper, D. Colonic butyrate-producing communities in humans: an overview using omics. Data. MSyst. 2, 1-18. (2017).

49. Koh, A., De Vadder, F., Kovatcheva-Datchary, P. \& Bäckhed, F. From dietary fiber to host physiology: short-chain fatty acids as key bacterial metabolites. Cell 165, 1332-1345 (2016).

50. Derrien, M. \& van Hylckama Vlieg, J. E. T. Fate, activity, and impact of ingested bacteria within the human gut microbiota. Trends Microbiol. 23, 354-366 (2015).

51. Dalile, B., Van Oudenhove, L., Vervliet, B. \& Verbeke, K. The role of short-chain fatty acids in microbiota-gut-brain communication. Nat. Rev. Gastroenterol. Hepatol. 16, 461-478 (2019).

52. Ait-Belgnaoui, A. et al. Prevention of gut leakiness by a probiotic treatment leads to attenuated HPA response to an acute psychological stress in rats. Psychoneuroendocrinology 37, 1885-95 (2012).

53. Elamin, E. E. et al. Short-chain fatty acids activate AMP-activated protein kinase and ameliorate ethanol-induced intestinal barrier dysfunction in Caco-2 cell monolayers. J. Nutr. 143, 1872-1881 (2013).

54. Scaldaferri, F. et al. The gut barrier: new acquisitions and therapeutic approaches. J. Clin. Gastroenterol. 46, 12-17 (2012).

55. Mohammadi, A. A. et al. The effects of probiotics on mental health and hypothalamic-pituitary-adrenal axis: a randomized, double-blind, placebocontrolled trial in petrochemical workers. Nutr. Neurosci. 19, 387-395 (2015).

56. Felger, J. C. \& Treadway, M. T. Inflammation effects on motivation and motor activity: role of dopamine. Neuropsychopharmacology 42, 216-241 (2017).

57. Rothhammer, V. et al. Type I interferons and microbial metabolites of tryptophan modulate astrocyte activity and central nervous system inflammation via the aryl hydrocarbon receptor. Nat. Med. 22, https://doi.org/10.1038/ $\mathrm{nm} .4106$ (2016)

58. Hao, Z., Wang, W., Guo, R. \& Liu, H. Faecalibacterium prausnitzii (ATCC 27766) has preventive and therapeutic effects on chronic unpredictable mild stressinduced depression-like and anxiety-like behavior in rats. Psychoneuroendocrinology 104, 132-142 (2019).

59. Cox, M. A. et al. Short-chain fatty acids act as antiinflammatory mediators by regulating prostaglandin E2 and cytokines. World J. Gastroenterol. 15, 5549-5557 (2009).

60. Vuholm, S., Lorenzen, J. K., Kristensen, M. Relative validity and reproducibility of a food frequency questionnaire to assess dietary fiber intake in Danish adults. Food Nutr. Res. 58, https://doi.org/10.3402/fnr.v58.24723 (2014).

61. Hudson, T. et al. Dietary fiber intake: assessing the degree of agreement between food frequency questionnaires and 4-day food records. J. Am. Coll. Nutr. 25, 370-381 (2006)

62. Bagga, D. et al. Influence of 4-week multi-strain probiotic administration on resting-state functional connectivity in healthy volunteers. Eur. J. Nutr. 58, 1821-1827 (2019).

63. Ji, B. W. et al. Quantifying spatiotemporal variability and noise in absolute microbiota abundances using replicate sampling Brian. Nat. Methods $\mathbf{1 6}$ 731-736 (2020).

64. Hugerth, L. W. \& Andersson, A. F. Analysing microbial community composition through amplicon sequencing: From sampling to hypothesis testing. Front Microbiol. 8, 1-22. (2017). 